speculum insertion and had perioperative hemodynamic stability. There was reduction in anaesthetic, analgesic, muscle relaxant consumption in group D. Emergence time and extubation time was less, and there were fewer episodes of postoperative nausea and vomiting and shivering in group D. VAS was less and time for first analgesic was prolonged in patients of dexmedetomidine group. There were few incidences of side-effects like bradycardia and hypotension. Conclusion: We conclude that dexmedetomidine as anesthetic adjuvant is efficacious in attenuating nasal speculum response, provides intraoperative hemodynamic stability, decreases intraoperative anaesthetic requirement and hastens early recovery without any serious side-effects.

\section{Miracle of a syringe attached to the pilot balloon of endotracheal tube during neurosurgical procedures}

\section{Karthik Raj, M. V. Niranjan Kumar, A. Rout \\ Department of Neuroanesthesia, Sri Ramachandra Medical College and Hospital, Porur, Chennai, Tamil Nadu, India}

Background: The use of cuff inflating syringe in situ results in preventing airway related complications during neurosurgical procedures in various positions. Materials and Methods: It is a prospective study including all neurological surgeries done under GA from 2000. The parameters noted were duration of anesthesia and a standard $10 \mathrm{ml}$ of air is used to inflate the cuff to prevent air leak, the remaining amount of air in syringe is noted and the amount of air left in situ is noted at the end of procedure. Results: In $20 \%$ of patients there was an increase in air volume of which 5-10\% increase was noted in $2 \mathrm{hrs}, 20-25 \%$ in $2-4 \mathrm{hrs}$ and up to $50 \%$ for surgeries lasting more than 5-6 hrs. We had 5 patients of which 3 patients who had air leak during posterior cervical surgeries and 2 patients had complete tube blockade at bevel end of the ET tube. Conclusion: Securing and maintaining the ET tube in long duration procedures and positions other than supine can be difficult as the patency of tube can be compromised also the added risk of using nitrous oxide which can diffuse into the cuff increasing its volume and pressure. Complications like tube blockade at bevel end, hoarseness, laryngeal edema, vocal cord edema, accidental extubation and ventilator malfunction can be prevented. The simple technique of leaving the cuff inflating syringe can help to avoid airway catastrophe as the airway is inaccessible during the procedure.

\section{Anesthesia for carotid stenting: Our experience}

\section{Deepa Chaturvedi}

Department of Anaesthesia, Neuroanesthesia unit, Max Super specialty hospital, Saket, New Delhi, India

Background: Patients with carotid artery disease frequently have other co-morbidities especially cardiac disease. A new option of treatment, carotid artery stenting (CAS) shows lower risk of stroke and myocardial infraction but high incidence of hemodynamic changes during procedure. We reviewed the anesthetic management and complications during and after treatment of CAS. Materials and Methods: One hundred and fourteen patients who had CAS over a period of four years from 2009-2013 were reviewed. For conventional common femoral artery approach procedure was done under conscious sedation with midazolam and fentanyl.Oxygen was supplied with nasal prongs. The groin site was anesthetized with local infiltration of lidocaine. Monitoring include ECG, invasive BP, SpO2. After femoral sheath insertion temporary pacemaker lead was placed and attached to the pacemaker. Prophylactic vagolytic agent atropine $0.6 \mathrm{mg}$ IV was used prior to balloon inflation to block the baroreceptor response. Heparin was given before placement of carotid stent. Result: Most of the patients were ASA Grade 3. Common pre-procedure morbidities were Coronary Artery Disease, HT, DM and peripheral vascular disease along with neurological deficits. 12 patients had TIA, 10 with stroke and 1 patient had aphasia. One patient had femoral artery tear at the puncture site, which was secured by Perclose-intra arterial suture. Conclusion: Anesthesia for carotid intervention requires protection of the brain from ischemic insult. Attention to physiological factors influencing cerebral blood flow is mandatory. The anesthesiologist plays a crucial role in maintaining hemodynamic stability, adjusting anticoagulation and monitoring neurological status.

\section{Comparison of hemodynamic responses to intubation: Flexible fibreoptic bronchoscope versus McCoy laryngoscope in presence of rigid cervical collar simulating cervical immobilization for traumatic cervical spine}

Nitesh Gill, Shobha Purohit

Department of Anaesthesia, S M S Medical College, Jaipur, Rajasthan, India

Background: Intubation is known to cause exaggerated hemodynamic response in form of tachycardia, hypertension and dysrrhythmias. In cervical spine immobility or instability, intubation has to be performed using cervical immobilization to prevent 
exacerbation of spinal cord injuries. Application of rigid cervical collar may reduce cervical spine movements, but it hinders tracheal intubation with standard laryngoscope. It significantly reduces the mouth opening, rendering laryngoscopy difficult and also lifts up the chin and tips the larynx anteriorly. The aim of this study is to compare the hemodynamic responses to fibreoptic bronchoscope and McCoy laryngoscope in patients undergoing elective surgery under general anesthesia with rigid cervical collar simulating cervical spine immobilization in the situation of cervical trauma. Materials and Methods: Thirty two patients in age range 20-50 years, of ASA I - $\Pi$, and of either sex undergoing elective surgery under general anesthesia were randomly allocated into each group. There were two groups according to the technique used for intubation: Group A (Flexible Fibreoptic Bronchoscope) and group B (McCoy Laryngoscope). Systolic Blood Pressure (SBP), Diastolic Blood Pressure (DBP), Mean arterial blood pressure (MAP) and heart rate (HR) were recorded at baseline, intra-operatively, immediately before and after induction, and immediately after intubation. Thereafter, every minute for the next five minutes. Demographic data is presented as numbers and intergroup comparison of these was done by Chi square test. The value of $P<0.05$ was considered statistically significant. Quantitative data is presented as mean values and standard deviation. Intergroup comparison of quantitative data was done by parametric test (unpaired $t$-test) and probability was considered to be significant if less than $<0.05$. Results: The demographic data is comparable. Due to intubation response, heart rate and blood pressure increased significantly $(P<0.05)$ above preoperative values in McCoy group as compared to fibreoptic group. Conclusion: We suggest that the flexible fibreoptic bronchoscope is an effective and better method of intubation in situation like traumatic cervical spine injury and provides stable hemodynamics.

\section{Attenuation of hemodynamic pressor response to laryngoscopy and intubation with single pre-induction dose of dexmedetomidine in patients undergoing neurosurgery and neurointervention}

\section{Neeta Karmarkar, Rajashree Gandhe}

Department of Anaesthesia, Kokilaben Dhirubhai Ambani Hospital, Mumbai, Maharashtra, India

Background: The aim of the study was to evaluate the effect of a single pre induction dose of dexmedetomidine on pressor response to laryngoscopy and intubation on patients undergoing neurosurgery and neurointervention and to assess the incidence of adverse effects of dexmedetomidine. Materials and Methods: After obtaining the approval of the hospital ethics committee an observational study was conducted on 100 ASA grade 1 and 2 patients. Written, informed valid consent was obtained from all patients. Inj. Dexmedetomidine was started in an infusion at the rate of $1 \mathrm{mcg} / \mathrm{kg}$ for ten minutes before induction. Heart rate and blood pressure were then recorded at specific intervals before and after intubation. Results: Sixty patients were enrolled for the study. The baseline heart rate was 77.15 (SD 14.49) beats per minute (bpm). One minute after intubation mean heart rate was 121.44 (SD 22.34) bpm. The baseline mean arterial pressure was 109.29 (SD 14.58) $\mathrm{mm} \mathrm{Hg}$. One minute after intubation mean value was 95.06 (SD 17.89) $\mathrm{mm} \mathrm{Hg}(P<0.001)$. The difference was significant in all parameters at the said intervals as compared to the baseline. No adverse effects were reported. Conclusion: Dexmedetomidine is a very useful drug as a premedicant @1 mcg/ $\mathrm{kg}$ in an infusion for ten minutes to attenuate the sympathetic response to laryngoscopy and intubation.

\section{A prospective randomized control evaluation of desflurane and propofol for emergence from anesthesia following resection of cerebellopontine angle tumors}

\section{Sohan Lal Solanki, Manohar Bhat, Hemant Bhagat ${ }^{1}$, Ishwar Bhukal ${ }^{1}$}

Departments of Anaesthesia, Critical Care and Pain, Tata Memorial Hospital, Mumbai, Maharashtra, ${ }^{1}$ Anaesthesia and Intensive Care, PGIMER, Chandigarh, India

Background:Desflurane and propofol are commonly used anesthetic agent in neurosurgical patients. Though these agents have been commonly used in patients undergoing supratentorial neurosurgery, there is paucity of data evaluating its use in surgery for cerebellopontine $(\mathrm{CP})$ angle tumors. The primary aim was to evaluate the time to emergence from anesthesia with use of desflurane when compared to propofol. The intraoperative hemodynamics, brain relaxation and vasomotor response during tumor resection were the secondary outcomes. Materials and Methods: The present study was a prospective randomized trial conducted on thirty adult patients undergoing $\mathrm{CP}$ angle tumor resection. Anesthesia was induced with morphine $0.1 \mathrm{mg} / \mathrm{kg}$ and thiopentone $4-6 \mathrm{mg} / \mathrm{kg}$ while muscle relaxation was achieved with vecuronium. Anesthesia was maintained with desflurane or propofol as per the randomization along with nitrous oxide (NO) to maintain a state 2 entropy value of 40-60. Desflurane or propofol were switched off after completion of skin sutures while NO was switched off following removal of skull pins. 